\title{
Aortic remodeling mismatch: A potential risk factor of late distal stent graft-induced new entry after frozen elephant trunk deployment
}

\author{
Takuya Wada, MD, Hiroshi Yamamoto, MD, PhD, Takayuki Kadohama, MD, PhD, and \\ Daichi Takagi, MD, PhD, Akita, Japan
}

\footnotetext{
From the Department of Cardiovascular Surgery, Akita University Graduate School of Medicine, Akita, Japan. Disclosures: The authors reported no conflicts of interest.

The Journal policy requires editors and reviewers to disclose conflicts of interest and to decline handling or reviewing manuscripts for which they may have a conflict of interest. The editors and reviewers of this article have no conflicts of interest.

Read at the 101st Annual Meeting of The American Association for Thoracic Surgery: A Virtual Learning Experience, April 30-May 2, 2021.

Received for publication Jan 11, 2021; accepted for publication April 12, 2021; available ahead of print June 18, 2021.

Address for reprints: Hiroshi Yamamoto, MD, PhD, Department of Cardiovascular Surgery, Akita University Graduate School of Medicine, Hondo 1-1-1, Akita, 010-8543 Japan (E-mail: h-yama@cvs.med.akita-u.ac.jp). JTCVS Techniques 2021;8:46-8

2666-2507

Copyright $(2021$ The Author(s). Published by Elsevier Inc. on behalf of The American Association for Thoracic Surgery. This is an open access article under the CC BY-NC-ND license (http://creativecommons.org/licenses/bync-nd/4.0/).

https://doi.org/10.1016/j.xjtc.2021.04.036
}

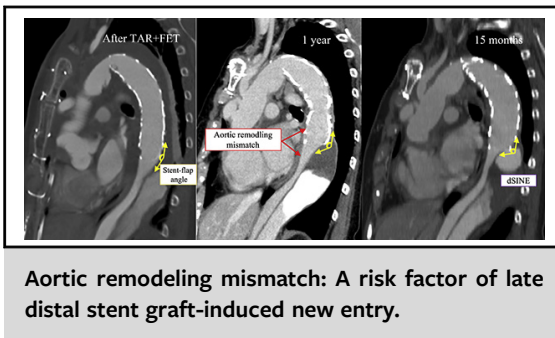

CENTRAL MESSAGE

Aortic remodeling mismatch between the stent-covered and nonstent-covered aorta may be a risk factor of late distal stent graft-induced new entry after frozen elephant trunk deployment.

See Commentaries on pages 49 and 51.
The mechanisms behind developing distal stent graftinduced new entry (dSINE) are complex. Stent graft oversizing, spring-back force, and pathological aortic wall fragility have been the main risk factors associated with dSINE. ${ }^{1}$ Nonetheless, the dSINE development mechanisms are still unclear because it is related to multiple factors. We report a case of postoperative dSINE that may have been caused by a mechanical stress at the distal edge of the deployed frozen elephant trunk (FET) as a result of steeper stent-flap angle between the stent-covered aorta and nonstent-covered aorta after total arch repair (TAR) with FET deployment for type A acute aortic dissection (TAAD).

\section{CASE REPORT}

A 64-year-old woman presented with sudden back pain. Computed tomography (CT) scan showed TAAD with a primary entry at the ascending aorta and a major reentry at the distal aortic arch (Figure 1, A). She underwent TAR with an FET using the zone 0 arch repair strategy. ${ }^{2}$ An FET graft ( $J$ Graft Frozenix; Japan Lifeline Co Ltd, Tokyo, Japan), a straight vascular prosthesis with a distal stented part (120$\mathrm{mm}$ stent length), was deployed from the trimmed distal aortic end (zone 0) toward the descending aorta. The FET graft's distal end was positioned at the eighth thoracic vertebrae level. The FET graft diameter $(27 \mathrm{~mm})$ was determined by the preoperative CT finding (90\% of the descending aortic diameter at the level of the main pulmonary artery).

The postoperative CT scan showed almost normalization of the true lumen (TL) diameter in the stent-covered aorta, whereas the persistent false lumen (FL) perfusion from the reentry tear was observed in the nonstent-covered downstream aorta (Figure 1, B). The follow-up CT scan 12 months after surgery revealed an upward shift (steeper angle to the FET long axis) of the flap, which was caused by a remodeling mismatch of the aortic dissecting wall in the distal transition zone between the stent-covered aorta and nonstent-covered aorta (Figure 1, C). The patient experienced back pain 15 months after surgery, and then CT scan revealed dSINE within the distal transition zone with further progression of the aortic remodeling mismatch (Figure 1, D, and Figure 2, A). She underwent urgent thoracic endovascular aortic repair (TEVAR). The 2 TEVAR stent-grafts were deployed in a retrograde fashion from the level proximal to the origin of the celiac artery 


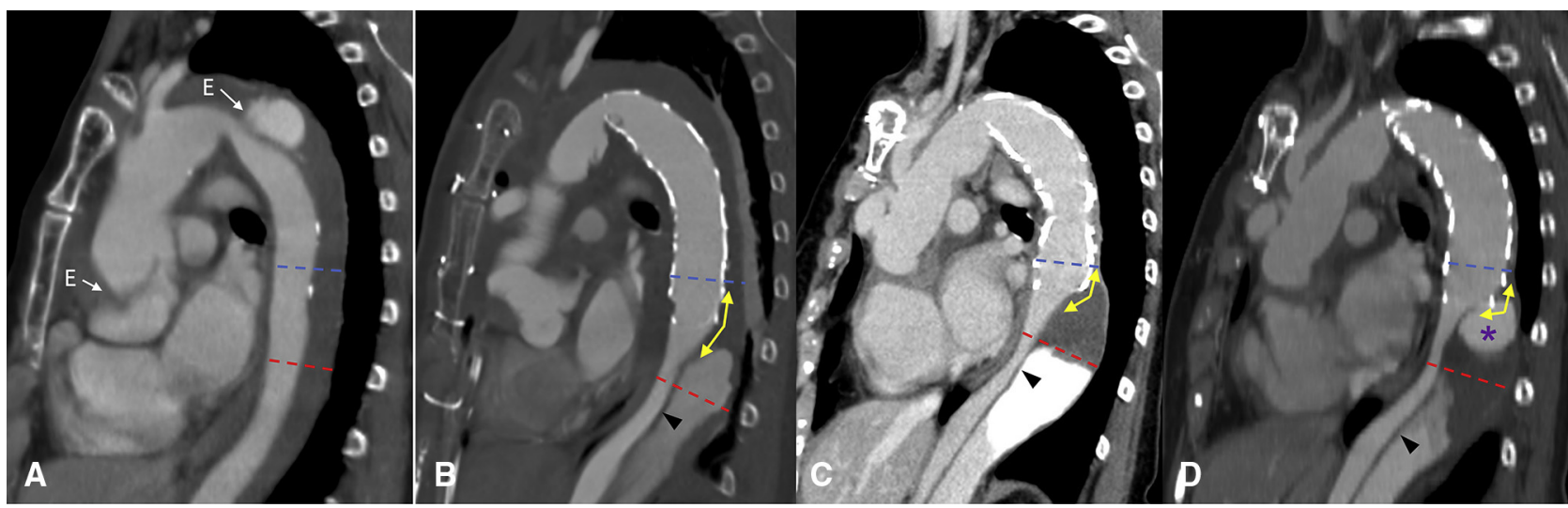

FIGURE 1. A time course of aortic configurations on enhanced computed tomography scans. A, At the onset of type A acute aortic dissection (TAAD). B, One week after total arch repair with a frozen elephant trunk (FET). C, Twelve months after surgery. D, Fifteen months after surgery, the onset of distal stentinduced new entry. The dotted lines indicate the levels at which the true and false lumen diameters were measured (blue line indicates $15 \mathrm{~mm}$ proximal to the distal end of the FET. Red line indicates $20 \mathrm{~mm}$ distal to the distal end of the FET). The angle between the 2 yellow arrows indicates the stent-flap angel. E, Entry tear (arrowhead, flap; purple asterisk, distal stent-induced new entry [flap perforation]).

to the inside of the FET graft, the first was Relay Plus endograft and the second was Relay Plus endograft (28/ $24 \times 150 \mathrm{~mm}$ and $30 \times 150 \mathrm{~mm}$, respectively) (Bolton Medical, Sunrise, Fla). The postoperative course was uneventful. Aortic-related complications were not observed over 2 years, and the follow-up CT scan showed a successful outcome (Figure 2, B).

The diameters of the TL and FL in the stent-covered aorta (proximal level) and nonstent-covered aorta (distal level) are shown in Table 1. The TL:FL increased at the proximal site but decreased at the distal site in a time-dependent manner (Table 1). The stent-flap angle in the distal transition zone steepened in a time-dependent manner (Table 1). Consent for this report was provided by the patient.

\section{DISCUSSION}

Recently, TAR with an FET for TAAD has been widely accepted because it is believed to promote aortic remodeling in the downstream aorta and reduce risks of reintervention. However, postoperative dSINE could be a life-threatening complication associated with FET

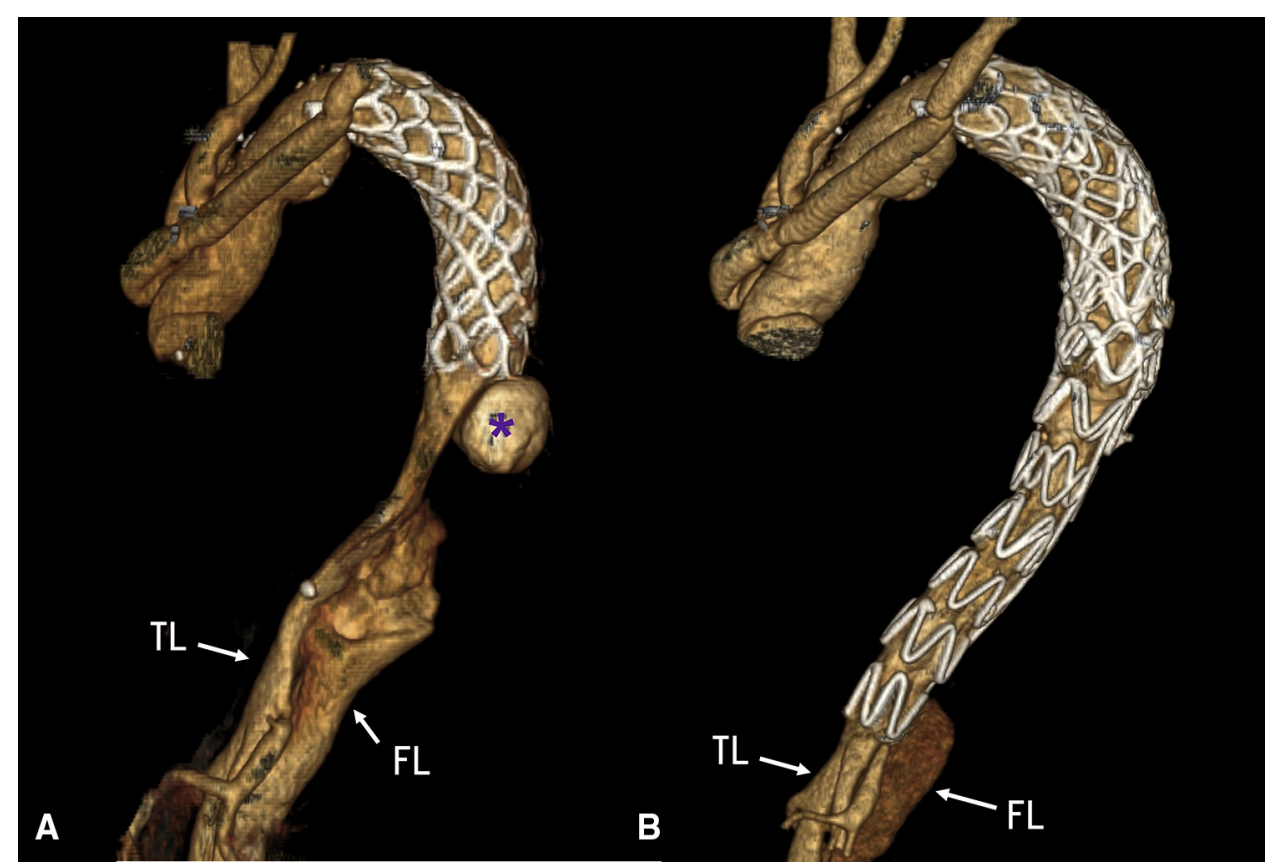

FIGURE 2. Three-dimensional computed tomography scans. A, At the onset of distal stent-induced new entry. B, After thoracic endovascular aortic repair treatment. $T L$, True lumen; $F L$, false lumen. *Distal stent-induced new entry. 
TABLE 1. The time course of true lumen (TL) diameter, false lumen (FL) diameter, TL to FL ratio at the proximal and distal levels, and stent-flap angle in the distal transition zone

\begin{tabular}{|c|c|c|c|c|c|}
\hline Level & Diameter/ratio & $\begin{array}{c}\text { A } \\
\text { TAAD onset }\end{array}$ & $\begin{array}{c}\text { B } \\
\text { After TAR/FET }\end{array}$ & $\begin{array}{c}\text { C } \\
\text { POM } 12\end{array}$ & $\begin{array}{c}\text { D } \\
\text { dSINE onset }\end{array}$ \\
\hline \multirow[t]{3}{*}{ Proximal } & $\mathrm{TL}(\mathrm{mm})$ & 15.5 & 21.4 & 26.2 & 27.6 \\
\hline & FL (mm) & 13.1 & 7.4 & 4.3 & 4.0 \\
\hline & TL:FL & 1.12 & 2.89 & 6.09 & 6.90 \\
\hline \multirow[t]{4}{*}{ Distal } & $\mathrm{TL}(\mathrm{mm})$ & 16.5 & 13.7 & 7.6 & 9.6 \\
\hline & $\mathrm{FL}(\mathrm{mm})$ & 14 & 22.1 & 35.0 & 45.0 \\
\hline & TL:FL ratio & 1.17 & 0.61 & 0.22 & 0.21 \\
\hline & Stent-flap angle (degree) & & 150 & 128 & 109 \\
\hline
\end{tabular}

TL and FL values in columns A, B, C, and D were the diameters measured in panels A, B, C, and D of Figure 1, respectively. Proximal and distal levels are corresponding to the blue and red dotted lines in Figure 1, respectively. Stent-flap angles were measured in panel A, B, C and D of Figure 1. Stent-flap angles are corresponding to the angles between the two yellow arrows in Figure 1. TAAD, Type A acute aortic dissection; TAR, total arch repair; FET, frozen elephant trunk; POM, postoperative months; $d S I N E$, distal stent graftinduced new entry.

procedures. The mechanism for dSINE development has been reported to include oversizing theory or spring-back force theory so far. In this report, we assessed the preoperative TL configuration to determine the oversizing (ie, radial force) effect of the used FET on dSINE development in the way described by Jang and colleagues, ${ }^{1}$ who investigated the parameters they called aortic taper ratio (preoperative assessment: 1-distal landing zone TL diameter/ proximal landing zone TL diameter) and oversizing ratio (postoperative assessment: stent graft diameter/distal landing TL diameter-1) in SINE development after TEVAR for type B aortic dissection. In our patient, aortic taper ratio and oversizing ratio was 0.66 and 058 , respectively, which was greater than the values in the SINE group reported by Jang and colleagues ${ }^{1}$ ( 0.32 and 0.47 , respectively), suggesting that oversizing at the distal end of the FET may be associated with the dSINE development. Spring-back force of the stent is a stress to the aortic wall caused by the spring force returning from curved to straight configuration, which is another mechanism of postoperative dSINEs. However, FET curvature did not change in our patient.

Stent-graft implantation brings about changes in the stent-covered aorta's biomechanical properties. A compliance mismatch between the stent-covered and nonstent-

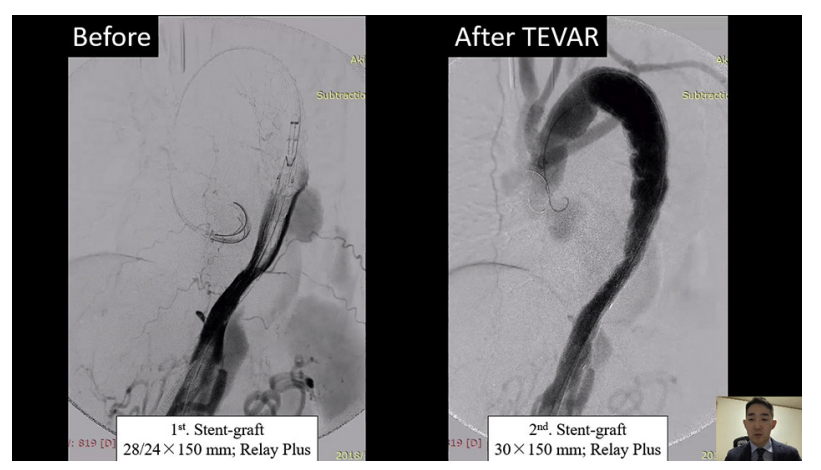

VIDEO 1. Case presentation. Video available at: https://www.jtcvs.org/ article/S2666-2507(21)00412-0/fulltext. covered aorta could have detrimental effects on stress and strain distributions. ${ }^{3}$ Although an FET has been reported to exert a beneficial effect on downstream aortic remodeling, ${ }^{4}$ the nonstent-covered aorta may be unable to undergo sufficient aortic remodeling due to FL expansion presumably caused by a retrograde flow and pulsatile wall stress in the FL. This could result in an upward shift on the flap (steeper angle to the aortic long axis) leading to a flap perforation (ie, dSINE) induced by mechanical stress at the distal edge of the deployed FET. This seems to be the most likely mechanism responsible for dSINE in our patient. Therefore, aortic remodeling mismatch in the distal transition zone between the stent-covered aorta and nonstent-covered aorta could be a potential risk factor of a late dSINE development following FET deployment in a patient with TAAD (Video 1).

Based on our experience, follow-up CT examinations at intervals $<1$ year may be recommended to detect the time-dependent change of aortic remodeling mismatch and predict dSINE development. Reinterventions for dSINEs can be successfully performed by using TEVAR techniques in a patient who underwent surgery for TAAD using an FET, because TEVARs are less invasive and eliminate flap perforations and residual tears. Further experience and investigation are needed to predict and optimally repair dSINEs.

\section{References}

1. Jang H, Kim MD, Kim GM, Won JY, Ko YG, Choi D, et al. Risk factors for stent graft-induced new entry after thoracic endovascular aortic repair for Stanford type B aortic dissection. J Vasc Surg. 2017;65:676-85.

2. Yamamoto H, Kadohama T, Yamaura G, Tanaka F, Takagi D, Kiryu K, et al. Total arch repair with frozen elephant trunk using the "zone 0 arch repair" strategy for type A acute aortic dissection. J Thorac Cardiovasc Surg. 2020;159:36-45.

3. Rinaudo A, Raffa GM, Scardulla F, Pilato M, Scardulla C, Pasta S. Biomechanical implications of excessive endograft protrusion into the aortic arch after thoracic endovascular repair. Comput Biol Med. 2015;66:235-41.

4. Di Bartolomeo R, Panteleo A, Berretta P, Murana G, Castrovinci S, Cefarelli M, et al. Frozen elephant trunk surgery in acute aortic dissection. J Thorac Cardiovasc Surg. 2015;149:S105-9. 